\title{
Biceps Femoris Activation during Hamstring Strength Exercises: A Systematic Review
}

\author{
Luis Llurda-Almuzara ${ }^{1,2,+(\mathbb{D})}$, Noé Labata-Lezaun ${ }^{1,2,+}(\mathbb{D})$, Carlos López-de-Celis 1,2,3 (D), \\ Ramón Aiguadé-Aiguadé ${ }^{4, * \mathbb{D}}$, Sergi Romaní-Sánchez ${ }^{1,2}$, Jacobo Rodríguez-Sanz ${ }^{1,2} \mathbb{D}^{\text {, }}$ \\ César Fernández-de-las-Peñas 5 (D) and Albert Pérez-Bellmunt 1,2,*(D)
}

check for updates

Citation: Llurda-Almuzara, L.; Labata-Lezaun, N.; López-de-Celis, C.; Aiguadé-Aiguadé, R.; Romaní-Sánchez, S.; Rodríguez-Sanz, J.; Fernández-de-las-Peñas, C.; Pérez-Bellmunt, A. Biceps Femoris Activation during Hamstring Strength Exercises: A Systematic Review. Int. J. Environ. Res. Public Health 2021, 18, 8733. https:// doi.org/10.3390/ijerph18168733

Academic Editor: Dustin Russel Slivka

Received: 7 July 2021

Accepted: 11 August 2021

Published: 18 August 2021

Publisher's Note: MDPI stays neutral with regard to jurisdictional claims in published maps and institutional affiliations.

Copyright: (c) 2021 by the authors. Licensee MDPI, Basel, Switzerland. This article is an open access article distributed under the terms and conditions of the Creative Commons Attribution (CC BY) license (https:// creativecommons.org/licenses/by/ $4.0 /)$
1 Faculty of Medicine and Health Sciences, Universitat Internacional de Catalunya, 08017 Sant Cugat del Vallès, Spain; 1llurda@uic.es (L.L.-A.); nlabata@uic.es (N.L.-L.); carlesldc@uic.es (C.L.-d.-C.); sergiromani@uic.es (S.R.-S.); jrodriguezs@uic.es (J.R.-S.)

2 ACTIUM Functional Anatomy Group, Universitat Internacional de Catalunya, 08195 Sant Cugat del Vallès, Spain

3 Institut Universitari per a la Recerca a I'Atenció Primària de Salut Jordi Gol i Gurina (IDIAPJGol), 08007 Barcelona, Spain

4 Department of Nursing and Physical Therapy, Universitat de Lleida, 25003 Lleida, Spain

5 Department of Physical Therapy, Occupational Therapy, Rehabilitation and Physical Medicine, Universidad Rey Juan Carlos, 28933 Madrid, Spain; cesar.fernandez@urjc.es

* Correspondence: raiguade@aiguade.com (R.A.-A.); aperez@uic.es (A.P.-B.); Tel.: +34-93-504-2014 (A.P.-B.)

+ Equal contribution to this work.

Abstract: Background: The aim of the study was to systematically evaluate the biceps femoris long head activation across cross-sectional hamstring strength exercise studies. Methods: A systematic review design was followed. The search strategy conducted in PubMed, Cochrane Library, and Web of Sciences databases found a total of 3643 studies. Once inclusion and exclusion criteria were applied, 29 studies were finally included in this systematic review. A total of 507 participants and 114 different exercises were analyzed. Exercises were evaluated individually and grouped into several categories: Nordics, isokinetic exercises, lunges, squats, deadlifts, good mornings, hip thrusts, bridges, leg curls, swings, hip and back extensions, and others. Results: Results showed the isokinetic and Nordic exercises as the categories with highest biceps femoris activation $(>60 \%$ of Maximal Voluntary Isometric Contraction). Nordic hamstring exercise ankle dorsiflexion was the exercise that achieved the highest biceps femoris long head activation (128.1\% of its Maximal Voluntary Isometric Contraction). Conclusions: The results from this systematic review suggest that isokinetic and Nordic exercises seem to be the best option to activate biceps femoris long head. Future studies evaluating the implementation of these exercises in prevention programs are needed.

Keywords: hamstring; muscle injury; biceps femoris; muscle activity; electromyography

\section{Introduction}

Hamstring strain injury (HSI) is one of the most common injuries in sports involving high-intensity sprinting, acceleration, and decelerations [1]. Injury rates of the hamstring muscles ranges from $6 \%$ to $29 \%$ of all injuries in track and field, soccer, Australian football, rugby, basketball, or cricket [2].

The biceps femoris long head (BFlh) is the most affected muscle, involving around $80 \%$ of all HSIs [3]. Moreover, around 30\% of HSIs are recurrent injuries [4]. In fact, previous HSI is a primary risk factor of a re-injury across literature [5,6]. Research into BFlh injuries continues to develop and has led to a better understanding of the problem [7].

Hamstring strength is one of the muscle properties that has received more attention in current research both as preventive and performance-enhancing strategy [8-10]. It seems that hamstring strength deficit is a good predictor of HSI [6]. In addition, strength has been also found to be a risk factor for preventing HSI in recent prospective cohort 
studies $[8,11,12]$. At the same time, hamstring strength seems to be positively correlated with athletic performance $[13,14]$. In fact, mostly all sport teams include hamstring strength exercises as part of their performance-enhancing and prevention strategy [15-18]. Improving athletic performance is a common objective in the sports field. The hamstring muscles are essential in many aspects of sports practice [18]. Hamstring muscle activity is higher than any other muscle group during a sport maneuver as essential to sport as sprinting [18]. For this reason, hamstring training exercises are a crucial component of sports performance.

Interestingly, there are a lot of strength exercises described and used in the current literature, making it difficult to determine the most appropriate when developing specific programs for the training of these muscles.

Several studies had compared BFlh muscle activation by using the surface electromyography (sEMG) activity level between hamstring strength exercises [19-21]. sEMG has been shown to be a good instrument to determine muscle activation levels during strength exercises [22]. sEMG has been used to categorize exercise intensity, and therefore, assisting sport coaches and physiotherapists when selecting the most appropriated exercise [21,23]. Previous studies have systematically reviewed muscle activation during commonly used strength exercises in the gluteus maximus (GMax) [24,25], which is another important hip extensor. However, no systematic review has previously investigated the differences in biceps femoris muscle activation across strength exercises. Therefore, the purpose of the current systematically review was to evaluate biceps femoris sEMG during the most common strengthening exercises in a healthy population.

\section{Materials and Methods}

\subsection{Registration}

The systematic review was performed in accordance with the Preferred Reporting Items for Systematic Reviews and Meta-Analyses (PRISMA) statement checklist [26]. The systematic review protocol was registered in the PROSPERO database with ID: CRD42020183079.

\subsection{Information Sources and Search}

The search strategy was developed following the PICO (Population, Intervention, Comparison, Outcomes) strategy.

Population: healthy adults without lower extremity injury;

Intervention: biceps femoris activation during hamstring strength exercises;

Comparison: no comparison due to specific cross-sectional study designs;

Outcome: biceps femoris muscle activity as assessed with sEMG was primary outcome. The "last 10 years" filter was used in all three databases. Keywords used to develop the search strategy are shown in Table 1.

Table 1. Keywords used for the search strategy.

\begin{tabular}{ccc}
\hline Exercise & Muscle Activity & Muscle \\
\hline Strength & Mucle development & Biceps femoris \\
Exercise & Myogenesis & Hamstring \\
Weight bearing & Myofibrillogenesis & Semitendinosus \\
Force & Hypertrophy & Semimembranosus \\
& Electromyography & Posterior thigh \\
& Excitation & Knee flexor \\
& Activation & Hip extensor \\
& EMG & \\
\hline
\end{tabular}

Databases used in the current systematic review were PubMed, Cochrane Library, and Web of Science. Furthermore, the lists of references from the studies included were checked 
to find other studies meeting inclusion criteria. The final search was performed on 1 May 2020. A complete PubMed database search strategy example is shown in Table 2.

Table 2. Example of search strategy in PubMed database.

(((((hamstring[Title/Abstract] OR biceps femoris[Title/Abstract] OR semitendinosus[Title/Abstract] OR semimembranosus[Title/Abstract] OR "posterior tight"[Title/Abstract] OR “knee flexors"[Title/Abstract] OR “hip extensors"[Title/Abstract])))

AND (("muscl* development"[Title/Abstract] OR myogenesis[Title/Abstract] OR myofibrillogenesis[Title/Abstract] OR hypertroph*[Title/Abstract] OR electromyogra*[Title/Abstract] OR excitation[Title/Abstract] OR emg[Title/Abstract] OR activity[Title/Abstract] OR activation[Title/Abstract]))) AND ((strengt*[Title/Abstract] OR exercis*[Title/Abstract] OR weight-bearing[Title/Abstract] OR force[Title/Abstract]))))

\subsection{Eligibility Criteria and Study Selection}

The inclusion criteria for studies included in this systematic review were as follows: (1) cross-sectional design; (2) healthy individuals; (3) evaluation of hamstring strength exercises; (4) providing data about sEMG of hamstring muscles; (5) providing data normalized by MVIC; (6) specific data about BFlh or "lateral hamstring musculature"; (7) English or Spanish language; and (8) published during the last 10 years. Studies were excluded if they included: (1) elderly people; (2) did not provide percentage activation data; or (3) did not specify the normalization method. Moreover, if studies provided data via bar charts, the corresponding author of the article was contacted, and the means and standard deviations of each exercise were requested.

Titles and abstracts were screened by two independent authors (SRS and LLL). In case of discrepancy, a third author (APB) was consulted. The Cohen's Kappa index was used in order to assess the inter-rater agreement. Landis et al. [27] categorized the Kappa Statistic as $<0.00$ as poor inter-rater agreement, $0.00-0.20$ as slight, $0.21-0.40$ as fair, $0.41-0.60$ as moderate, $0.61-0.80$ as substantial, and $0.81-1.00$ as almost perfect inter-rater agreement.

\subsection{Data Collection Process}

The following data were extracted for studies included in this systematic review: (1) Author's last name and year of publication; (2) sample size; (3) exercises performed; (4) normalization method; (5) electrode placement; and (6) testing load.

\subsection{Outcomes}

As primary outcome, the average sEMG root mean square (RMS) expressed as a percentage of MVIC for BFlh /lateral hamstring musculature was chosen.

This review categorized hamstring exercises according to muscle activation of the hamstring muscles following Macadam and Feser [28]: low activation (0 to 20\% Maximal Voluntary Isometric Contraction, MVIC), moderate activation (21 to 40\% MVIC), high activation (41 to $60 \%$ MVIC), or very high activation (61\% or greater MVIC).

\subsection{Risk of Bias of Individual Studies}

The National Institutes of Health Quality Assessment Tool for Observational Cohort and Cross-sectional Studies was used to assess the methodology quality of the included studies [29]. This tool contains 14 quality questions assessing internal validity (population, sample size, statistical analysis, and outcome measures). Questions must be answered as "Yes", "No", "cannot determine (CD)", "not applicable (NA)", or "not reported (NR)". Cross-sectional studies automatically scored "not applicable" on criteria 6, 7 and 10 (Supplementary Table S1). One point was obtained only if the question was answered as "Yes". All questions were equally weighted in overall quality assessment results. 


\section{Results}

\subsection{Study Selection}

The search strategy found a total of 3643 studies (PubMed: 987; ScienceDirect: 2083; Cochrane Library: 573). Two thousand six hundred and twenty studies were initially included after checking for duplicates. After title screening, 291 studies were considered for full abstract screening. One hundred and forty-two studies were excluded after reading the abstract, so 143 full-text articles were assessed for eligibility. Full-text screening was carried out, and 43 studies finally met the inclusion criteria. The reasons for the exclusion of 106 studies were as follows: did not provide data as \%MVIC $(n=8)$, did not assess hamstring strength exercises $(n=47)$, did not include healthy participants $(n=6)$, did not specify sEMG normalization method $(n=24)$, did not provide isolated BFlh/lateral hamstring data $(n=14)$, no cross-sectional design $(n=2)$, others $(n=5)$. Finally, 43 studies were included in the qualitative analysis. From these 43, twenty did not provide numerical data other than bar charts. Thus, the corresponding authors from these papers were contacted. Twenty-nine studies were finally included in the quantitative analysis. The Cohen's Kappa index showed an "almost perfect" agreement $(\mathrm{k}=0.87)$. The detailed study selection and reasons for excluded articles can be found in the PRISMA flow chart (Figure 1).

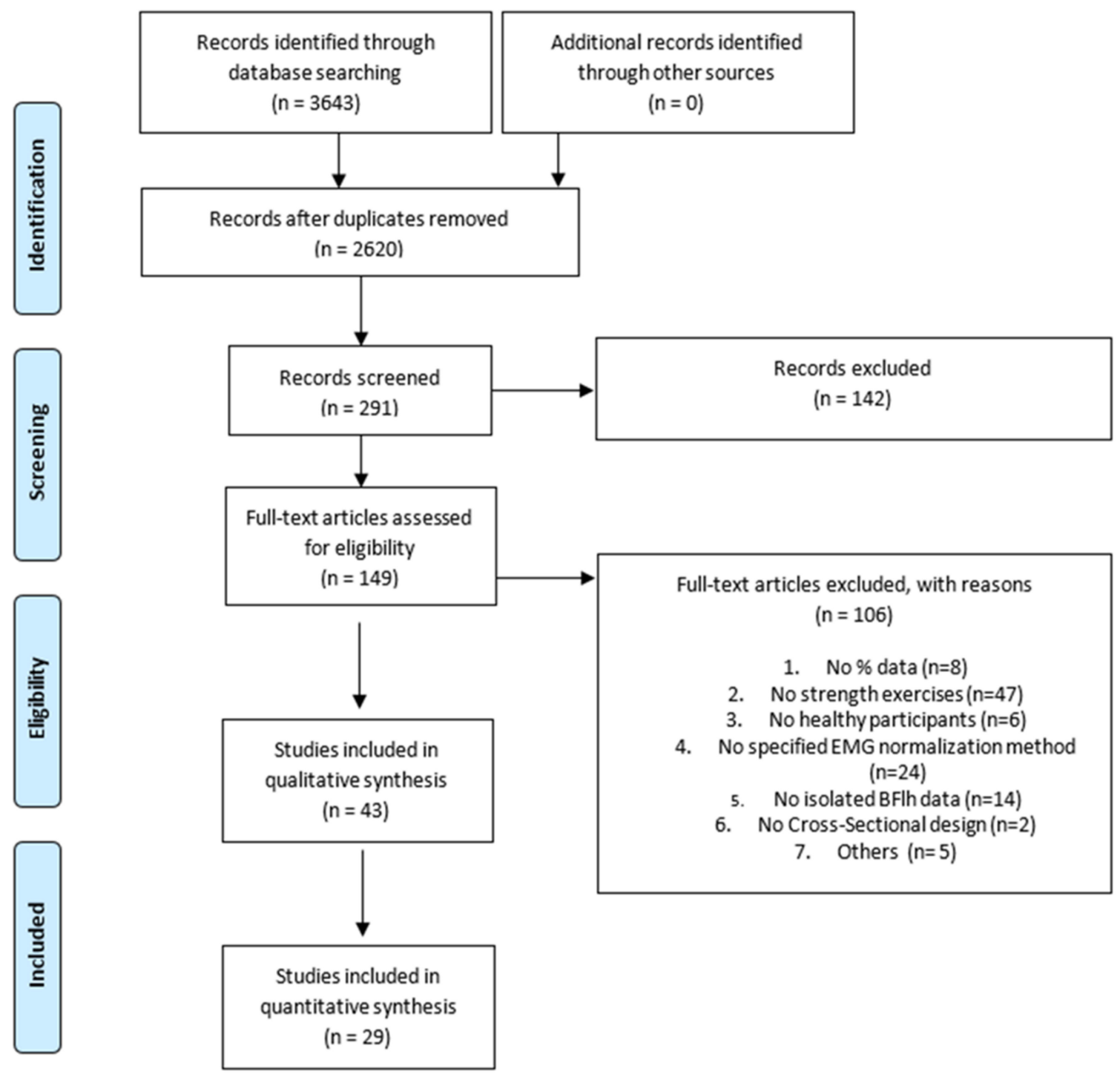

Figure 1. Preferred Reporting Items for Systematic Reviews and Meta Analyses (PRISMA) flow diagram. 


\subsection{Study Characteristics}

Studies involved a total of 507 participants with an age ranging from 19 to 30 years. The exercises mostly commonly assessed in the studies were swings, dead lifts, Nordic hamstring exercises (NHE), squats, leg curls, lunges, good mornings, hip thrusts, and hip extensions. The majority of the studies normalized hamstring EMG data by using MVC in prone position with the knee flexed at $45^{\circ}$ and the sEMG electrodes placed following the Surface Electromyography for the Non-Invasive Assessment of Muscles (SENIAM) guideline [30].

The testing load differed among studies, but bodyweight was the more commonly used load. Table 3 summarizes the characteristics of the included studies. 
Table 3. Characteristics of included exercises.

\begin{tabular}{|c|c|c|c|c|c|c|c|}
\hline Study & $\begin{array}{l}\text { Quality } \\
\text { Score }\end{array}$ & $\begin{array}{l}\text { Sample Size } \\
\text { (Age) }\end{array}$ & Exercises (Category) & Exercises (Detailed) & $\begin{array}{l}\text { Normalization } \\
\text { Method }\end{array}$ & Electrode Placement & Testing Load \\
\hline Jeon 2016 [31] & 6 & $16(23.4 \pm 2.2)$ & Hip extensions & $\begin{array}{l}\text { Prone hip extension } \\
\text { Prone table hip extension } \\
\text { Prone table hip extension } \\
\text { with knee flexion }\end{array}$ & $\begin{array}{l}\text { According to the } \\
\text { guidelines of } \\
\text { Kendall et al. [32] }\end{array}$ & $\begin{array}{l}70 \% \text { on the line } \\
\text { extending between the } \\
\text { ischial tuberosity and } \\
\text { lateral epicondyle }\end{array}$ & No specified \\
\hline $\begin{array}{l}\text { Del Monte } \\
2020[33]\end{array}$ & 6 & $14(30 \pm 3.9)$ & Swing & $\begin{array}{c}\text { Squat swing } \\
\text { Hip hinge swing } \\
\text { Double knee extension } \\
\text { swing }\end{array}$ & $\begin{array}{l}\text { Prone position with } \\
\text { the knee flexed to } 90^{\circ}\end{array}$ & $\begin{array}{l}\text { According to SENIAM } \\
\text { guidelines }\end{array}$ & $\begin{array}{l}\text { Maximum mass the } \\
\text { participant could swing for a } \\
\text { cadence of } \\
35-40 \text { repetitions } / \mathrm{min} \\
\text { during the participant's } \\
\text { typical training sessions and } \\
\text { ranged from } \\
16 \text { to } 48 \mathrm{~kg}\end{array}$ \\
\hline $\begin{array}{c}\text { Lyons } \\
2017[34]\end{array}$ & 6 & $\begin{array}{c}14 \\
(21.5 \pm 2.03)\end{array}$ & Swing & $\begin{array}{c}\text { Swing } \\
\text { Snatch swing } \\
\text { Clean swing }\end{array}$ & $\begin{array}{l}\text { Prone position with } \\
\text { the knee flexed to } 70^{\circ}\end{array}$ & $\begin{array}{l}\text { Lateral aspect of the } \\
\text { thigh } 67 \% \text { of the distance } \\
\text { between the trochanter } \\
\text { and popliteal fossa, } \\
\text { starting at the trochanter }\end{array}$ & $\begin{array}{l}\text { Load for each individual } \\
\text { exercise that could be } \\
\text { performed for } \\
\text { 8-10 repetitions with a good } \\
\text { technique. It ranged from } \\
4.5 \text { to } 32 \mathrm{~kg}\end{array}$ \\
\hline $\begin{array}{c}\text { Monajati } \\
2017[35,36]\end{array}$ & 5 & $10(22 \pm 4.7)$ & $\begin{array}{l}\text { Nordic Hamstring Exercise } \\
\text { Ball leg curl }\end{array}$ & $\begin{array}{c}\text { Nordic hamstring exercise } \\
\text { Leg ball curl }\end{array}$ & $\begin{array}{l}\text { Prone position with } \\
\text { the knee flexed to } 45^{\circ}\end{array}$ & $\begin{array}{l}\text { According to SENIAM } \\
\text { guidelines }\end{array}$ & Bodyweight \\
\hline $\begin{array}{l}\text { Lehecka } \\
2017[36]\end{array}$ & 4 & $\begin{array}{c}28 \\
(23.43 \pm 2.28)\end{array}$ & Bridge & $\begin{array}{l}\text { Single-leg bridges different } \\
\text { positions }\end{array}$ & $\begin{array}{l}\text { Prone position with } \\
\text { the knee flexed to } 45^{\circ}\end{array}$ & $\begin{array}{l}\text { According to SENIAM } \\
\text { guidelines }\end{array}$ & Bodyweight \\
\hline $\begin{array}{l}\text { Marshall } \\
2010[37]\end{array}$ & 5 & $14(24.1 \pm 1.7)$ & $\begin{array}{l}\text { Others } \\
\text { Hip extensions } \\
\text { Bridges }\end{array}$ & $\begin{array}{c}\text { Swiss ball rolls } \\
\text { Swiss ball hip extension } \\
\text { Swiss ball praying mantis } \\
\text { Swiss ball single leg squat } \\
\text { Prone hold } \\
\text { Swiss Ball hold and crunch } \\
\text { Swiss ball bridge }\end{array}$ & No specified & No specified & Bodyweight \\
\hline
\end{tabular}


Table 3. Cont.

\begin{tabular}{|c|c|c|c|c|c|c|c|}
\hline Study & $\begin{array}{l}\text { Quality } \\
\text { Score }\end{array}$ & $\begin{array}{l}\text { Sample Size } \\
\text { (Age) }\end{array}$ & Exercises (Category) & Exercises (Detailed) & $\begin{array}{c}\text { Normalization } \\
\text { Method }\end{array}$ & Electrode Placement & Testing Load \\
\hline $\begin{array}{c}\text { Khaiyat } 2018 \\
{[38]}\end{array}$ & 4 & $\begin{array}{c}12 \\
(20.10 \pm 1.10)\end{array}$ & $\begin{array}{l}\text { Lunges } \\
\text { Others } \\
\text { Bridge } \\
\text { Squats }\end{array}$ & $\begin{array}{l}\text { Double-leg raise } \\
\text { Forward lunge } \\
\text { Glute bridge } \\
\text { Sit-up } \\
\text { Squat }\end{array}$ & $\begin{array}{l}\text { Prone position with } \\
\text { the knee flexed to } 45^{\circ}\end{array}$ & $\begin{array}{l}\text { According to SENIAM } \\
\text { guidelines }\end{array}$ & Bodyweight \\
\hline $\begin{array}{l}\text { Arias-Poblete } \\
2019 \text { [39] }\end{array}$ & 6 & $\begin{array}{c}30 \\
(21.8 \pm 1.46)\end{array}$ & $\begin{array}{c}\text { Deadlifts } \\
\text { Swings } \\
\text { Nordic Hamstring } \\
\text { Exercises } \\
\text { Bridges } \\
\text { Others }\end{array}$ & $\begin{array}{c}\text { Single-leg deadlift } \\
\text { Swing } \\
\text { Nordic hamstring exercise } \\
\text { Bridge on chair } \\
\text { Prone bridge } \\
\text { Bridge in lateral position } \\
\text { Strike } \\
\text { Neutral back bridge } \\
\text { Slip leg } \\
\text { Heel strike against ball } \\
\text { Four supports with } \\
\text { extended arms and legs } \\
\text { Scissors held in lateral } \\
\text { position } \\
\text { Single bridge }\end{array}$ & No specified & $\begin{array}{l}\text { According to SENIAM } \\
\text { guidelines }\end{array}$ & Bodyweight \\
\hline $\begin{array}{l}\text { Collazo } \\
2020[40]\end{array}$ & 5 & $7(29.4 \pm 4.6)$ & Hip thrusts & $\begin{array}{c}\text { Hip thrust } \\
\text { Pull hip thrust } \\
\text { Rotation hip thrust } \\
\text { Feet-away hip thrust }\end{array}$ & $\begin{array}{l}\text { Prone position with } \\
\text { the knee flexed to } 45^{\circ}\end{array}$ & $\begin{array}{l}\text { According to SENIAM } \\
\text { guidelines }\end{array}$ & $40 \% 1 \mathrm{RM}$ \\
\hline $\begin{array}{l}\text { Contreras } \\
2016[41]\end{array}$ & 4 & $13(28.9 \pm 5.1)$ & Hip thrusts & $\begin{array}{l}\text { Barbell hip thrust } \\
\text { Band hip thrust } \\
\text { American hip thrust }\end{array}$ & $\begin{array}{l}\text { Prone position with } \\
\text { the knee flexed to } 45^{\circ}\end{array}$ & $\begin{array}{l}\text { According to SENIAM } \\
\text { guidelines }\end{array}$ & $10 \mathrm{RM}$ \\
\hline $\begin{array}{l}\text { Severini } \\
2018[42]\end{array}$ & 5 & $\begin{array}{c}11 \\
(22.2 \pm 1.38)\end{array}$ & $\begin{array}{c}\text { Deadlifts } \\
\text { Others } \\
\text { Hip extension }\end{array}$ & $\begin{array}{l}\text { Extender } \\
\text { Diver } \\
\text { Glider }\end{array}$ & Knee flexion & $\begin{array}{l}\text { According to SENIAM } \\
\text { guidelines }\end{array}$ & Bodyweight \\
\hline
\end{tabular}


Table 3. Cont.

\begin{tabular}{|c|c|c|c|c|c|c|c|}
\hline Study & $\begin{array}{l}\text { Quality } \\
\text { Score }\end{array}$ & $\begin{array}{l}\text { Sample Size } \\
\text { (Age) }\end{array}$ & Exercises (Category) & Exercises (Detailed) & $\begin{array}{c}\text { Normalization } \\
\text { Method }\end{array}$ & Electrode Placement & Testing Load \\
\hline $\begin{array}{c}\text { Mausehund } \\
2018 \text { [43] }\end{array}$ & 5 & $13(24.9 \pm 2.9)$ & Squats & $\begin{array}{c}\text { Rear foot elevated split squat } \\
\text { Single-leg squat } \\
\text { Split squat }\end{array}$ & $\begin{array}{l}\text { Prone position with } \\
\text { the knee flexed to } 45^{\circ}\end{array}$ & $\begin{array}{l}\text { According to SENIAM } \\
\text { guidelines }\end{array}$ & 6-8 RM \\
\hline $\begin{array}{l}\text { Hegyi } \\
2019[19]\end{array}$ & 6 & $19(26.1 \pm 3.2)$ & $\begin{array}{l}\text { Deadlifts } \\
\text { Hip extensions } \\
\text { Good morning } \\
\text { Leg curl } \\
\text { Bridges }\end{array}$ & $\begin{array}{c}\text { Straight-knee bridge } \\
\text { Upright hip extension } \\
\text { conic-pulley } \\
\text { Slide leg curl } \\
\text { Prone leg curl } \\
45^{\circ} \text { hip extension } \\
\text { Bent-knee bridge } \\
\text { Cable pendulum } \\
\text { Unilateral Romanian } \\
\text { deadlift } \\
\text { Good morning }\end{array}$ & $\begin{array}{l}\text { Lay prone with the } \\
\text { trunk and hip fixed } \\
\text { to the dynamometer } \\
\text { bench in neutral } \\
\text { position }\end{array}$ & $\begin{array}{l}\text { Midpoint along the } \\
\text { ischial } \\
\text { tuberosity-popliteal } \\
\text { fossa distance }\end{array}$ & $12 \mathrm{RM}$ \\
\hline Vigotsky 2015 [44] & 6 & $15(24.6 \pm 5.3)$ & Good morning & Good morning & $\begin{array}{l}\text { Prone position with } \\
\text { the knee flexed to } 45^{\circ}\end{array}$ & $\begin{array}{l}\text { On the muscle bellies, } \\
\text { parallel with muscle } \\
\text { fibers }\end{array}$ & Submaximal $1 \mathrm{RM}$ \\
\hline $\begin{array}{l}\text { Lawrence } \\
2019 \text { [45] }\end{array}$ & 7 & $20(26.8 \pm 7.8)$ & Back extension & $\begin{array}{c}\text { Back extension } \\
\text { Reverse hyperextension }\end{array}$ & $\begin{array}{l}\text { While the subject was } \\
\text { in the top position of } \\
\text { hip extension }\end{array}$ & $\begin{array}{l}\text { According to SENIAM } \\
\text { guidelines }\end{array}$ & No specified \\
\hline Kim 2013 [46] & 7 & $\begin{array}{c}22 \\
(23.5 \pm 4.92)\end{array}$ & Hip extension & $\begin{array}{l}\text { Floor hip extension } \\
\text { Round foam roll hip } \\
\text { extension }\end{array}$ & No specified & $\begin{array}{l}2 \mathrm{~cm} \text { from the lateral } \\
\text { border of the thigh and } \\
\text { two-thirds of the } \\
\text { distance between the } \\
\text { trochanter and the back } \\
\text { of the knee }\end{array}$ & No specified \\
\hline
\end{tabular}


Table 3. Cont

\begin{tabular}{|c|c|c|c|c|c|c|c|}
\hline Study & $\begin{array}{l}\text { Quality } \\
\text { Score }\end{array}$ & $\begin{array}{c}\text { Sample Size } \\
\text { (Age) }\end{array}$ & Exercises (Category) & Exercises (Detailed) & $\begin{array}{l}\text { Normalization } \\
\text { Method }\end{array}$ & Electrode Placement & Testing Load \\
\hline Jeon 2017 [47] & 8 & $16(25.4 \pm 4.2)$ & Hip extension & $\begin{array}{l}\text { Prone table hip extension } \\
\text { Prone table hip extension } \\
\text { with abdominal drawing-in } \\
\text { Prone table hip extension } \\
\text { with the abdominal } \\
\text { drawing-in maneuver with } \\
\text { the flexed contralateral knee } \\
\text { joint on a chair }\end{array}$ & $\begin{array}{l}\text { According to the } \\
\text { guidelines of } \\
\text { Kendall et al. [33] }\end{array}$ & $\begin{array}{l}\text { Two-thirds of the } \\
\text { distance along the line } \\
\text { extending between the } \\
\text { ischial tuberosity and } \\
\text { lateral epicondyle }\end{array}$ & Bodyweight \\
\hline Kawama 2020 [48] & 6 & $14(19.6 \pm 1.0)$ & Deadlift & $\begin{array}{c}\text { Adduction double-leg } \\
\text { deadlift } \\
\text { Neutral double-leg deadlift } \\
\text { Abduction double-leg } \\
\text { deadlift } \\
20^{\circ} \text { internal rotation } \\
\text { double-leg deadlift } \\
20^{\circ} \text { External rotation } \\
\text { double-leg deadlift } \\
40^{\circ} \text { External rotation } \\
\text { double-leg deadlift }\end{array}$ & Knee flexion & $\begin{array}{l}\text { Over } 40 \text { and } 60 \% \text { of the } \\
\text { thigh length (the } \\
\text { distance between the } \\
\text { greater trochanter }(0 \%) \\
\text { and the popliteal crease } \\
(100 \%)) \text { for BFlh }\end{array}$ & $60 \%$ of their body mass \\
\hline Ryu 2012 [49] & 6 & $\begin{array}{c}14 \\
(23.3 \pm 3.74)\end{array}$ & Bridge & $\begin{array}{l}\text { Bridge on stable base } \\
\text { Bridge on unstable base }\end{array}$ & No specified & $\begin{array}{l}\text { According to SENIAM } \\
\text { guidelines }\end{array}$ & Bodyweight \\
\hline Choi 2016 [50] & 6 & $27(27.8 \pm 5.8)$ & Bridge & $\begin{array}{c}\text { Bridge } \\
\text { Single bridge } \\
\text { Single bridge with hip } \\
\text { abduction } \\
\text { Single bridge with sling } \\
\text { Single bridge with sling and } \\
\text { hip abduction }\end{array}$ & No specified & $\begin{array}{l}\text { On the thigh between the } \\
\text { knee and buttocks }\end{array}$ & Bodyweight \\
\hline
\end{tabular}


Table 3. Cont.

\begin{tabular}{|c|c|c|c|c|c|c|c|}
\hline Study & $\begin{array}{l}\text { Quality } \\
\text { Score }\end{array}$ & $\begin{array}{l}\text { Sample Size } \\
\text { (Age) }\end{array}$ & Exercises (Category) & Exercises (Detailed) & $\begin{array}{l}\text { Normalization } \\
\text { Method }\end{array}$ & Electrode Placement & Testing Load \\
\hline Lee 2019 [51] & 5 & $\begin{array}{c}26 \\
(23.15 \pm 2.68)\end{array}$ & Hip extension & $\begin{array}{l}\text { Prone hip extension } \\
\text { Prone hip extension with hip } \\
\text { abduction and knee flexion }\end{array}$ & $\begin{array}{l}\text { According to the } \\
\text { guidelines of } \\
\text { Kendall et al. [33] }\end{array}$ & $\begin{array}{l}2 \mathrm{~cm} \text { from the lateral } \\
\text { border of the thigh and } \\
\text { two-thirds of the } \\
\text { distance between the } \\
\text { trochanter and the back } \\
\text { of the knee }\end{array}$ & Bodyweight \\
\hline $\begin{array}{c}\text { Comfort } 2017 \\
\text { [52] }\end{array}$ & 6 & $15(22.6 \pm 2.1)$ & Nordic hamstring exercise & $\begin{array}{c}\text { Nordic hamstring ankle } \\
\text { dorsiflexed } \\
\text { Nordic hamstring ankle } \\
\text { plantar flexed }\end{array}$ & $\begin{array}{l}\text { Prone position with } \\
\text { the knee flexed to } 45^{\circ}\end{array}$ & $\begin{array}{l}\text { Placed at the midline of } \\
\text { the muscle belly of both } \\
\text { the BF }\end{array}$ & Bodyweight maximal effort \\
\hline Park 2019 [53] & 7 & 21 (NR) & Nordic hamstring exercise & $\begin{array}{l}10^{\circ} \text { Nordic hamstring base } \\
\text { slope angle } 0^{\circ} \\
10^{\circ} \text { Nordic hamstring base } \\
\text { slope angle } 10^{\circ} \\
10^{\circ} \text { Nordic hamstring base } \\
\text { slope angle } 15^{\circ} \\
15^{\circ} \text { Nordic hamstring base } \\
\text { slope angle } 0^{\circ} \\
15^{\circ} \text { Nordic hamstring base } \\
\text { slope angle } 10^{\circ} \\
15^{\circ} \text { Nordic hamstring base } \\
\text { slope angle } 15^{\circ}\end{array}$ & No specified & $\begin{array}{l}\text { Two-thirds of the } \\
\text { distance between the } \\
\text { trochanter and the back } \\
\text { of the knee }\end{array}$ & Bodyweight maximal effort \\
\hline $\begin{array}{l}\text { Muyor } \\
2020[54]\end{array}$ & 6 & $20(24 \pm 5.55)$ & $\begin{array}{l}\text { Squat } \\
\text { Lunge }\end{array}$ & $\begin{array}{l}\text { Monopodal squat } \\
\text { Forward lunge } \\
\text { Lateral step-up }\end{array}$ & $\begin{array}{l}\text { Prone position with } \\
\text { the knee flexed to } 45^{\circ}\end{array}$ & $\begin{array}{l}\text { According to SENIAM } \\
\text { guidelines }\end{array}$ & $60 \%$ of $5 \mathrm{RM}$ \\
\hline $\begin{array}{l}\text { Jónasson } \\
2016 \text { [55] }\end{array}$ & 7 & $40(24.1 \pm 2.6)$ & Isokinetic & $\begin{array}{c}\text { Isometric knee flexion } \\
\text { medial rotation } \\
\text { Isometric knee flexion lateral } \\
\text { rotation }\end{array}$ & $\begin{array}{l}\text { Prone position with } \\
\text { the knee flexed to } 45^{\circ}\end{array}$ & $\begin{array}{l}\text { According to SENIAM } \\
\text { guidelines }\end{array}$ & $5 \mathrm{~s}$ isometric \\
\hline
\end{tabular}


Table 3. Cont

\begin{tabular}{|c|c|c|c|c|c|c|c|}
\hline Study & $\begin{array}{l}\text { Quality } \\
\text { Score }\end{array}$ & $\begin{array}{c}\text { Sample Size } \\
\text { (Age) }\end{array}$ & Exercises (Category) & Exercises (Detailed) & $\begin{array}{l}\text { Normalization } \\
\text { Method }\end{array}$ & Electrode Placement & Testing Load \\
\hline Park 2014 [56] & 6 & $\begin{array}{l}20(21.94 \pm \\
2.24)\end{array}$ & Back extension & $\begin{array}{c}\text { Back extension, knee } \\
\text { extended, hands on sternum } \\
\text { Back extension, knee } \\
\text { extended, hands behind } \\
\text { head } \\
\text { Back extension, knee flexed, } \\
\text { hands on sternum } \\
\text { Back extension, knee flexed, } \\
\text { hands behind head }\end{array}$ & $\begin{array}{l}\text { Prone position with } \\
\text { the knee flexed to } 45^{\circ}\end{array}$ & $\begin{array}{l}\text { At the muscle in the } \\
\text { center of the back of the } \\
\text { thigh, approximately } \\
\text { half the distance from } \\
\text { the gluteal fold to the } \\
\text { back of the leg }\end{array}$ & Bodyweight \\
\hline $\begin{array}{c}\text { Contreras } \\
2016[57]\end{array}$ & 6 & $13(28.9 \pm 5.1)$ & Squats & $\begin{array}{c}\text { Front squat } \\
\text { Full squat } \\
\text { Parallell squat }\end{array}$ & $\begin{array}{l}\text { Prone position with } \\
\text { the knee flexed to } 45^{\circ}\end{array}$ & $\begin{array}{l}\text { According to SENIAM } \\
\text { guidelines }\end{array}$ & $10 \mathrm{RM}$ \\
\hline $\begin{array}{c}\text { Narouei } 2018 \\
{[58]}\end{array}$ & 6 & $\begin{array}{c}10(26.1 \pm \\
5.46)\end{array}$ & Nordic hamstring exercise & Nordic hamstring exercise & No specified & $\begin{array}{c}\text { According to SENIAM } \\
\text { guidelines }\end{array}$ & Maximal effort \\
\hline $\begin{array}{c}\text { Andersen } \\
2018 \text { [59] }\end{array}$ & 5 & $13(21.9 \pm 1.6)$ & $\begin{array}{l}\text { Hip thrusts } \\
\text { Deadlift }\end{array}$ & $\begin{array}{c}\text { Hip thrust } \\
\text { Deadlift } \\
\text { Hex bar deadlift }\end{array}$ & $\begin{array}{l}\text { Prone position with } \\
\text { the knee flexed to } 45^{\circ}\end{array}$ & $\begin{array}{c}\text { According to SENIAM } \\
\text { guidelines }\end{array}$ & $1 \mathrm{RM}$ \\
\hline
\end{tabular}




\subsection{Quality Assessment}

The studies included in this systematic review showed a mean score of 5.75 from a total of 11 points (from 4 to 8 points) in the National Institutes of Health Quality Assessment Tool for Observational Cohort and Cross-sectional Studies [29].

\subsection{Muscle Activation}

A total of 114 exercises were assessed across articles included in this review. Two different evaluations were performed across exercises. Firstly, we assessed them and ordered them from lower to higher biceps femoris activation (based on \%MVIC). Secondly, as many studies performed similar exercises, we grouped them into the following categories: Nordics, deadlifts, hip thrust, swing, squats, good mornings, bridges, hip extensions, isokinetic exercises, and lunges (Figure 2). Data regarding the 114 exercises are available in Supplementary Table S2.

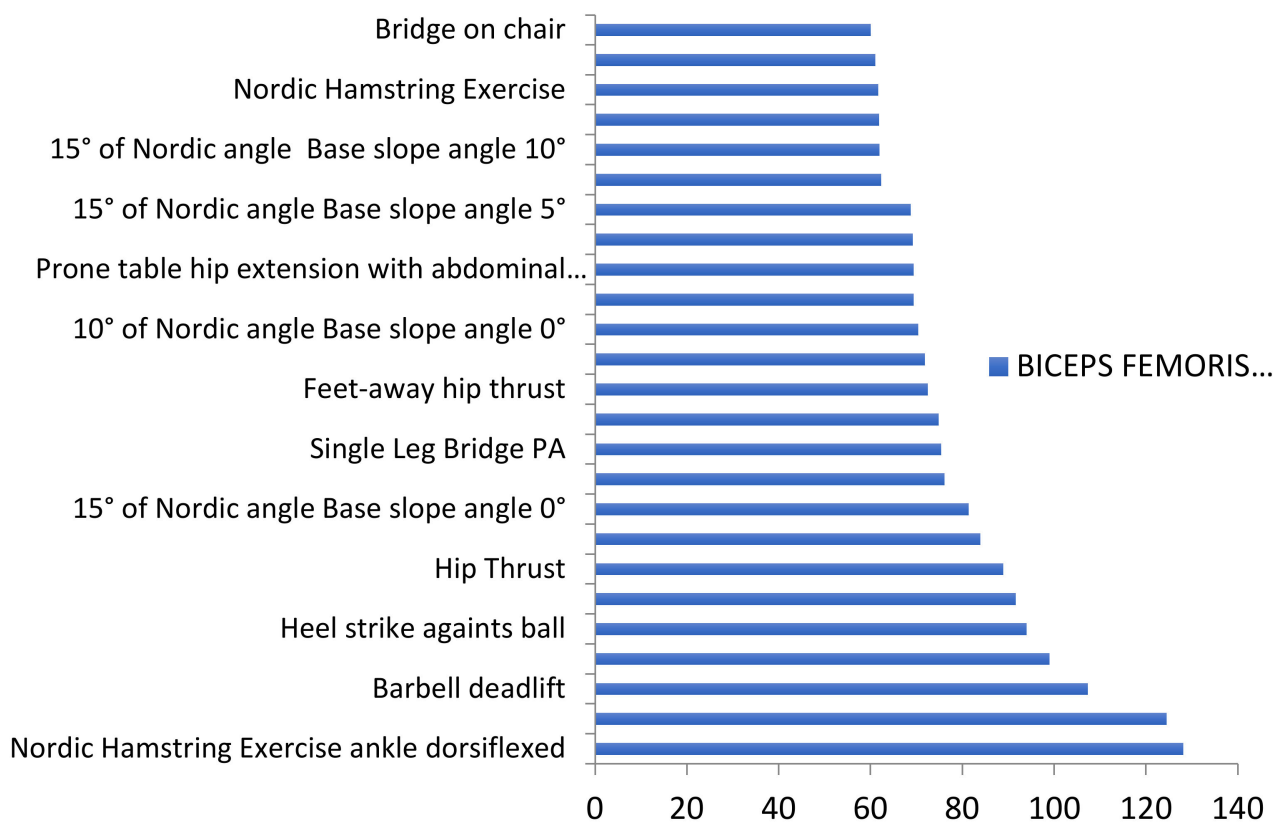

Figure 2. Exercises with very high BF activation levels.

This figure highlights both NHEs from Comfort et al. [52] and barbell deadlifts from Andersen et al. [59] with biceps femoris activation higher than 100\% MVIC. Moreover, the slip leg exercise and the heel strike against ball exercise from Arias-Poblete et al. [39] achieved $99 \%$ and $94 \%$ of BFlh activation, respectively.

The assessment of categories instead of exercises gives more global information about the exercise typology. Moreover, it minimizes a possible bias in one study which could notably alter the results. Figure 3 shows the results of BFlh activation levels by categories.

Very high activation ( $>60 \%$ MVIC).

Isokinetic exercises represented the highest biceps femoris activation with a mean of $81.7 \%$ MVIC. However, only two exercises were able to be included in this category. Furthermore, NHE also achieved a very high mean activation of 76.5\%MVIC. In this second case, eleven NHEs were evaluated. All of them achieved a mean activation higher than $60 \%$ MVIC. In fact, the NHE with ankle dorsiflexion achieved the higher BFlh activation in this systematic review with $128 \%$ MVIC.

High activation (from $41 \%$ to $60 \%$ MVIC).

Three exercise categories showed a mean activation between $41 \%$ and $60 \%$ of the MVIC: hip thrust (53.50\%), leg curl (48.78\%), and deadlifts (42.17\%).

Moderate activation (from $21 \%$ to $40 \%$ MVIC). 
Swing exercises $(40.52 \%)$, hip or back extensions (38.19\%), glute bridges $(32.34 \%)$, squats $(30.24 \%)$, other exercises $(28.09 \%)$, and good mornings $(22.79 \%)$ were the six categories that produced a moderate activation of the BFlh muscle.

Low activation $(<20 \%$ MVIC).

Only the lunge exercise category achieved a mean activation lower than $20 \%$. This category showed a mean BFlh activation level of $19.82 \%$ of MVIC.

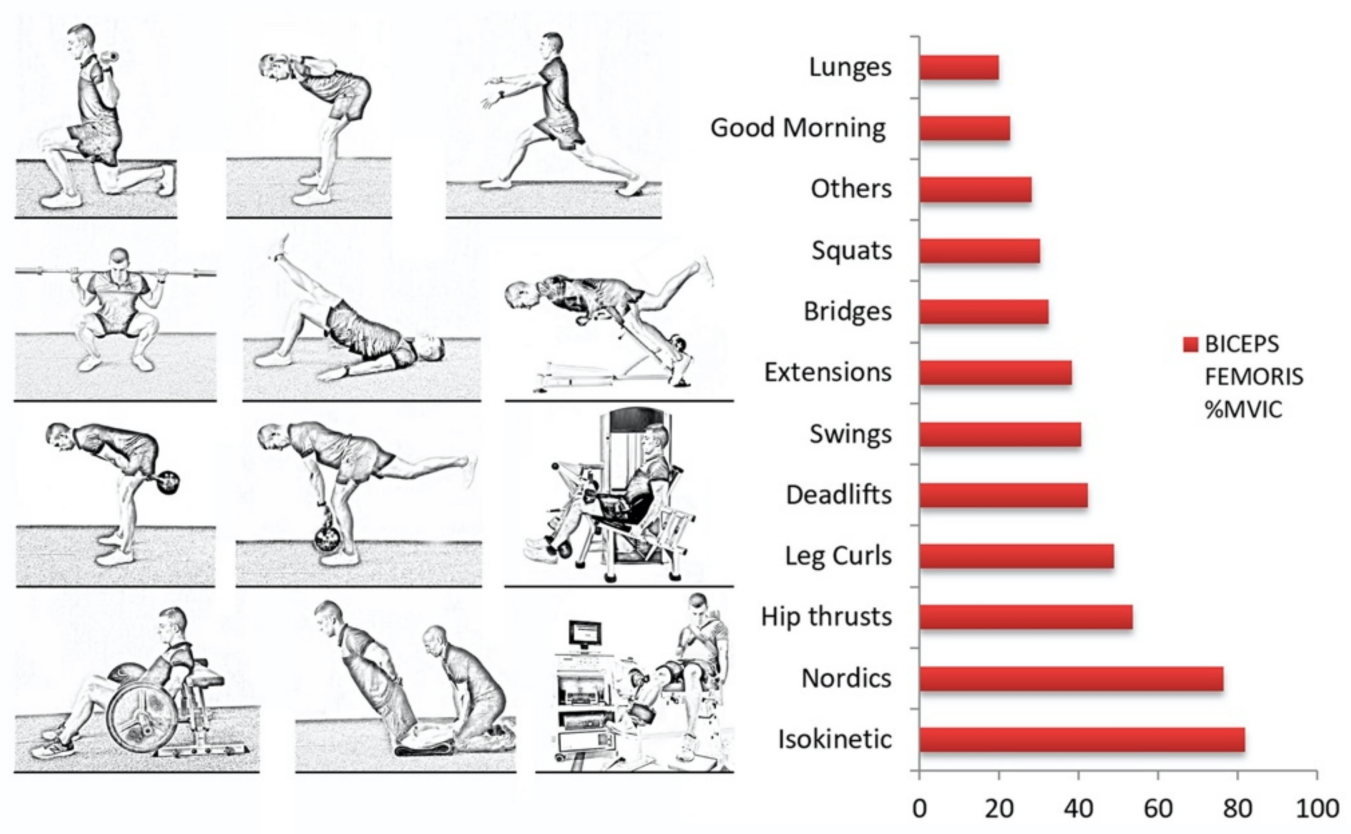

Figure 3. Categories ordered by BF mean activation levels.

\section{Discussion}

This systematic review aimed to evaluate the biceps femoris activation among different strength hamstring exercises. The results have shown that the Nordic hamstring exercise with ankle dorsiflexion is the exercise achieving the highest BFlh muscle activation. Moreover, the Nordic hamstring category (all variations) is the second-best exercise for BG activation category. These results may assist coaches, practitioners, or physiotherapists in selecting exercises based on intensity purposes.

The Nordic hamstring exercise has been widely used in the literature. Its first use in research was 2004 when Mjølsnes et al. [60] evaluated its effectiveness in hamstring eccentric strength. The use of NHE to improve hamstring eccentric strength is undoubtable. Several publications [61-63] and a recent systematic review and meta-analysis [64] support this idea. Furthermore, the meta-analyses from van Dyk et al. [65] and Al Attar et al. [66] concluded that including the NHE as an injury prevention exercise reduces the rate of HSI. Although some authors have questioned its use [67], current and previous data support the NHE as being associated with the highest levels of muscle BFlh activation.

The isokinetic exercise category was the highest in BFlh activation of all the categories. Isokinetic exercises have been commonly used for testing, for predicting injury risk, and as criteria for return to play after both hamstring and anterior cruciate ligament injury $[6,68,69]$. However, results from this study also support its use as a potential form of hamstring strengthening due to its very high activation of the biceps femoris muscle.

The results from the two exercises included in the "others" category also need attention. The "slip leg" and "heel strike against ball" exercises are quite different from deadlifts, swings, thrusts, curls, etc. However, they have shown activation levels of $94 \%$ and $99 \%$ of MVIC, placing them at the top fourth and fifth position in the ranking. Thus, these results would recommend these exercises as part of high-intensity hamstring training. 
On the other hand, lunges were the lowest BFlh activation category. These results are due to the fact that lunge exercises are commonly used for improving gluteus muscle but not hamstring strength. In fact, a systematic review evaluating gluteus maximus activation during common strength exercises found lunges variations as high activity ( $>60 \%$ of MVIC) exercises [24]. Thus, this kind of exercise should not be performed when hamstring activation and strength is the objective of the program.

This review focuses on muscle activation during different exercises commonly used to improve sport performance. However, other parameters such as strength, timing, or position may play an important role when choosing exercises [6,70,71]. Malliaropoulus et al. [6] supported the idea that hamstring strength exercises should also be oriented to specific sport demands. The highest activation of the biceps femoris occurs during the late swing phase and early stance phase of high speed running [71]. Thus, both open and closed kinetic chain exercises should be supported to reproduce the high demands of sprinting during sport maneuvers. Furthermore, hamstrings present a dual role in both hip and knee joints at the same time while running [72]. For this reason, both hip and knee dominant exercises should be supported to "simulate" their function during sprinting [6,73].

Although muscle activation gains are usually understood to be a prediction of strength gains, this relationship has not been scientifically proven [70]. This difference between muscle activation and strength must be taken into account when interpreting current results. Nevertheless, it should be also considered how this muscle is integrated in relation to other synergistic muscles involved in the particular or specific movement pattern [70].

\subsection{Limitations}

We should recognize some limitations from this systematic review. First, some studies evaluating the impact of exercise selection on muscle activity had to be excluded because they did not normalize their sEMG data by MVIC test. Second, in this systematic review, we did not consider exercise loads due to the high heterogeneity and the fact that it could alter the results. The high variability in the normalization methods makes difficult to compare directly several studies. Finally, current data are based only on healthy people; we do not currently know if muscle activation of injured sports players would exhibit a similar tendency. Furthermore, this review did not specify whether the participants were professional athletes or amateurs, nor did it specify the type of sport practiced. Therefore, it cannot be assured that these results can be extrapolated to a specific sport or to a specific level of sport expertise.

\subsection{Sports and Clinical Applications}

The strength training BFlh programs must be effective and specific to the necessity of sports athletes. The effective BFlh training programs are mandatory to enhance athletic performance. Exercise selection plays an important role to achieve this. This systematic review provides evidence regarding which exercises activate more the biceps femoris, which is one of the main muscles in sports. The results have shown that Nordic hamstring exercises and isokinetic exercises were more efficient to activate the BFlh more.

\section{Conclusions}

The results from this systematic review allow coaches, athletic trainers, and physical therapists to classify different exercises from low to high muscle activation of the BFlh. These data could implement a progressive training strategy to improve hamstrings strength and therefore athletic performance. However, although this review involves data from more than five hundred participants, individual anatomy variations could modify muscle activations during exercises. Thus, an anamnesis and physical examination of each participant is always recommended in order to individualize exercise selection.

Supplementary Materials: The following are available online at https:/ / www.mdpi.com/article/10 .3390/ijerph18168733/s1, Table S1: Risk of Bias of Individual Studies and Table S2: Muscle Activation. 
Author Contributions: Conceptualization, A.P.-B. and L.L.-A.; methodology, N.L.-L., S.R.-S. and J.R.-S.; formal analysis, L.L.-A., C.F.-d.-1.-P. and C.L.-d.-C.; writing—original draft preparation, A.P.-B., C.F.-d.-1.-P. and L.L.-A.; writing-review and editing, N.L.-L., R.A.-A., C.L.-d.-C., S.R.-S. and J.R.-S. All authors have read and agreed to the published version of the manuscript.

Funding: This research received no external funding.

Institutional Review Board Statement: Not applicable.

Informed Consent Statement: Not applicable.

Conflicts of Interest: The authors declare no conflict of interest.

\section{References}

1. Erickson, L.N.; Sherry, M.A. Rehabilitation and return to sport after hamstring strain injury. J. Sport Health Sci. 2017, 6, 262-270. [CrossRef]

2. Mendiguchia, J.; Alentorn-Geli, E.; Brughelli, M. Hamstring strain injuries: Are we heading in the right direction? Br. J. Sports Med. 2012, 46, 81-85. [CrossRef] [PubMed]

3. Bourne, M.N.; Opar, D.A.; Williams, M.D.; Al Najjar, A.; Shield, A.J. Muscle activation patterns in the Nordic hamstring exercise: Impact of prior strain injury. Scand. J. Med. Sci. Sports 2016, 26, 666-674. [CrossRef]

4. Dalton, S.L.; Kerr, Z.Y.; Dompier, T.P. Epidemiology of Hamstring Strains in 25 NCAA Sports in the 2009-2010 to 2013-2014 Academic Years. Am. J. Sports Med. 2015, 43, 2671-2679. [CrossRef] [PubMed]

5. Freckleton, G.; Pizzari, T. Risk factors for hamstring muscle strain injury in sport: A systematic review and meta-analysis. Br. J. Sports Med. 2013, 47, 351-358. [CrossRef]

6. Lee, J.W.Y.; Mok, K.-M.; Chan, H.C.K.; Yung, P.S.H.; Chan, K.-M. Eccentric hamstring strength deficit and poor hamstring-toquadriceps ratio are risk factors for hamstring strain injury in football: A prospective study of 146 professional players. J. Sci. Med. Sport 2018, 21, 789-793. [CrossRef] [PubMed]

7. Pérez-Bellmunt, A.; Miguel-Pérez, M.; Brugué, M.B.; Cabús, J.B.; Casals, M.; Martinoli, C.; Kuisma, R. An anatomical and histological study of the structures surrounding the proximal attachment of the hamstring muscles. Man. Ther. 2015, 20, 445-450. [CrossRef]

8. Maniar, N.; Shield, A.J.; Williams, M.D.; Timmins, R.G.; Opar, D.A. Hamstring strength and flexibility after hamstring strain injury: A systematic review and meta-analysis. Br. J. Sports Med. 2016, 50, 909-920. [CrossRef]

9. Opar, D.A.; Williams, M.D.; Shield, A.J. Hamstring strain injuries: Factors that lead to injury and re-injury. Sports Med. 2012, 42, 209-226. [CrossRef]

10. Opar, D.A.; Williams, M.D.; Timmins, R.G.; Hickey, J.; Duhig, S.J.; Shield, A.J. Eccentric Hamstring Strength and Hamstring Injury Risk in Australian Footballers. Med. Sci. Sports Exerc. 2015, 47, 857-865. [CrossRef]

11. Opar, D.A.; Williams, M.D.; Timmins, R.G.; Dear, N.M.; Shield, A.J. Knee flexor strength and bicep femoris electromyographical activity is lower in previously strained hamstrings. J. Electromyogr. Kinesiol. Off. J. Int. Soc. Electrophysiol. Kinesiol. 2013, 23, 696-703. [CrossRef]

12. Timmins, R.G.; Bourne, M.N.; Shield, A.J.; Williams, M.D.; Lorenzen, C.; Opar, D.A. Short biceps femoris fascicles and eccentric knee flexor weakness increase the risk of hamstring injury in elite football (soccer): A prospective cohort study. Br. J. Sports Med. 2016, 50, 1524-1535. [CrossRef]

13. Markovic, G.; Sarabon, N.; Boban, F.; Zoric, I.; Jelcic, M.; Sos, K.; Scappaticci, M. Nordic Hamstring Strength of Highly Trained Youth Football Players and Its Relation to Sprint Performance. J. Strength Cond. Res. 2020, 34, 800-807. [CrossRef]

14. Ishøi, L.; Aagaard, P.; Nielsen, M.F.; Thornton, K.B.; Krommes, K.K.; Hölmich, P.; Thorborg, K. The Influence of Hamstring Muscle Peak Torque and Rate of Torque Development for Sprinting Performance in Football Players: A Cross-Sectional Study. Int. J. Sports Physiol. Perform. 2019, 14, 665-673. [CrossRef]

15. O'Brien, J.; Finch, C.F. Injury prevention exercise programmes in professional youth soccer: Understanding the perceptions of programme deliverers. BMJ Open Sport Exerc. Med. 2016, 2, e000075. [CrossRef] [PubMed]

16. Read, P.J.; Jimenez, P.; Oliver, J.L.; Lloyd, R.S. Injury prevention in male youth soccer: Current practices and perceptions of practitioners working at elite English academies. J. Sports Sci. 2018, 36, 1423-1431. [CrossRef] [PubMed]

17. Emery, C.A.; Roy, T.-O.; Whittaker, J.L.; Nettel-Aguirre, A.; van Mechelen, W. Neuromuscular training injury prevention strategies in youth sport: A systematic review and meta-analysis. Br. J. Sports Med. 2015, 49, 865-870. [CrossRef] [PubMed]

18. Morin, J.-B.; Gimenez, P.; Edouard, P.; Arnal, P.; Jimenez-Reyes, P.; Samozino, P.; Brughelli, M.; Mendiguchia, J. Sprint Acceleration Mechanics: The Major Role of Hamstrings in Horizontal Force Production. Front. Physiol. 2015, 6, 404. [CrossRef] [PubMed]

19. Hegyi, A.; Csala, D.; Péter, A.; Finni, T.; Cronin, N.J. High-density electromyography activity in various hamstring exercises. Scand. J. Med. Sci. Sports 2019, 29, 34-43. [CrossRef] [PubMed]

20. McCurdy, K.; Walker, J.; Yuen, D. Gluteus Maximus and Hamstring Activation During Selected Weight-Bearing Resistance Exercises. J. Strength Cond. Res. 2018, 32, 594-601. [CrossRef]

21. Bourne, M.N.; Williams, M.D.; Opar, D.A.; Al Najjar, A.; Kerr, G.K.; Shield, A.J. Impact of exercise selection on hamstring muscle activation. Br. J. Sports Med. 2017, 51, 1021-1028. [CrossRef] 
22. Enoka, R.M.; Duchateau, J. Inappropriate interpretation of surface EMG signals and muscle fiber characteristics impedes understanding of the control of neuromuscular function. J. Appl. Physiol. 2015, 119, 1516-1518. [CrossRef]

23. Bourne, M.N.; Timmins, R.G.; Opar, D.A.; Pizzari, T.; Ruddy, J.D.; Sims, C.; Williams, M.D.; Shield, A.J. An Evidence-Based Framework for Strengthening Exercises to Prevent Hamstring Injury. Sports Med. 2018, 48, 251-267. [CrossRef]

24. Neto, W.K.; Soares, E.G.; Vieira, T.L.; Aguiar, R.; Chola, T.A.; Sampaio, V.; de Lima Sampaio, V.; Gama, E.F. Gluteus Maximus Activation during Common Strength and Hypertrophy Exercises: A Systematic Review. J. Sports Sci. Med. 2020, 19, 195-203.

25. Reiman, M.P.; Bolgla, L.A.; Loudon, J.K. A literature review of studies evaluating gluteus maximus and gluteus medius activation during rehabilitation exercises. Physiother. Theory Pract. 2012, 28, 257-268. [CrossRef]

26. Moher, D.; Liberati, A.; Tetzlaff, J.; Altman, D.G.; Altman, D.; Antes, G.; Atkins, D.; Barbour, V.; Barrowman, N.; Berlin, J.A.; et al. Preferred reporting items for systematic reviews and meta-analyses: The PRISMA statement. PLoS Med. 2009, 6, e1000097. [CrossRef]

27. Landis, J.R.; Koch, G.G. The Measurement of Observer Agreement for Categorical Data. Biometrics 1977, 33, 159. [CrossRef] [PubMed]

28. Macadam, P.; Feser, E.H. Examination of Gluteus Maximus Electromyographyic Excitation Associated with Dynamic Hip Extension during Body Weight Exercise: A Systematic Review. Int. J. Sports Phys. Ther. 2019, 14, 14-31. [CrossRef] [PubMed]

29. Ma, L.-L.; Wang, Y.-Y.; Yang, Z.-H.; Huang, D.; Weng, H.; Zeng, X.-T. Methodological quality (risk of bias) assessment tools for primary and secondary medical studies: What are they and which is better? Mil. Med. Res. 2020, 7, 7. [CrossRef] [PubMed]

30. Hermens, H.J.; Freriks, B.; Disselhorst-Klug, C.; Rau, G. Development of recommendations for SEMG sensors and sensor placement procedures. J. Electromyogr. Kinesiol. 2000, 10, 361-374. [CrossRef]

31. Jeon, I.; Hwang, U.; Jung, S.; Kwon, O. Comparison of gluteus maximus and hamstring electromyographic activity and lumbopelvic motion during three different prone hip extension exercises in healthy volunteers. Phys. Ther. Sport 2016, $22,35-40$. [CrossRef] [PubMed]

32. Kendall, F.P.; McCreary, E.K.; Provance, P.G. Muscle Testing and Function, 5th ed.; Williams \& Wilkins: Baltimore, MD, USA, 2005.

33. Del Monte, M.J.; Opar, D.A.; Timmins, R.G.; Ross, J.A.; Keogh, J.W.; Lorenzen, C. Hamstring Myoelectrical Activity During Three Different Kettlebell Swing Exercises. J. Strength Cond. Res. 2020, 34, 1953-1958. [CrossRef] [PubMed]

34. Lyons, B.C.; Mayo, J.J.; Tucker, W.S.; Wax, B.; Hendrix, R.C. Electromyographical Comparison of Muscle Activation Patterns Across Three Commonly Performed Kettlebell Exercises. J. Strength Cond. Res. 2017, 31, 2363-2370. [CrossRef] [PubMed]

35. Monajati, A.; Larumbe-Zabala, E.; Goss-Sampson, M.; Naclerio, F. Analysis of the Hamstring Muscle Activation During two Injury Prevention Exercises. J. Hum. Kinet. 2017, 60, 29-37. [CrossRef]

36. Lehecka, B.J.; Edwards, M.; Haverkamp, R.; Martin, L.; Porter, K.; Thach, K.; Sack, R.J.; Hakansson, N.A. Building a Better Gluteal Bridge: Electromyographyic Analysis of Hip Muscle Activity during Modified Single-leg Bridges. Int. J. Sports Phys. Ther. 2017, 12, 543-549.

37. Marshall, P.W.M.; Desai, I. Electromyographic analysis of upper body, lower body, and abdominal muscles during advanced Swiss ball exercises. J. Strength Cond. Res. 2010, 24, 1537-1545. [CrossRef]

38. Khaiyat, O.A.; Norris, J. Electromyographic activity of selected trunk, core, and thigh muscles in commonly used exercises for ACL rehabilitation. J. Phys. Ther. Sci. 2018, 30, 642-648. [CrossRef]

39. Arias-Poblete, L.; Álvarez-Zúñiga, M.; Contreras-Diaz, G.; Jerez-Mayorga, D.; Antúnez, V.J.; Monteverde Sánchez, A. Classification of strengthening exercises of the lower member posterior chain, based on the amplitude of muscular activation in amateur soccer players between 18-25 years. J. Sport Health Res. 2019, 11, 155-163.

40. Collazo García, C.L.; Rueda, J.; Suárez Luginick, B.; Navarro, E. Differences in the Electromyographic Activity of Lower-Body Muscles in Hip Thrust Variations. J. Strength Cond. Res. 2020, 34, 2449-2455. [CrossRef]

41. Contreras, B.; Vigotsky, A.D.; Schoenfeld, B.J.; Beardsley, C.; Cronin, J. A Comparison of Gluteus Maximus, Biceps Femoris, and Vastus Lateralis Electromyography Amplitude for the Barbell, Band, and American Hip Thrust Variations. J. Appl. Biomech. 2016, 32, 254-260. [CrossRef]

42. Severini, G.; Holland, D.; Drumgoole, A.; Delahunt, E.; Ditroilo, M. Kinematic and electromyographic analysis of the Askling L-Protocol for hamstring training. Scand. J. Med. Sci. Sports 2018, 28, 2536-2546. [CrossRef]

43. Mausehund, L.; Skard, A.E.; Krosshaug, T. Muscle Activation in Unilateral Barbell Exercises: Implications for Strength Training and Rehabilitation. J. Strength Cond. Res. 2019, 33, S85-S94. [CrossRef] [PubMed]

44. Vigotsky, A.D.; Harper, E.N.; Ryan, D.R.; Contreras, B. Effects of load on good morning kinematics and EMG activity. PeerJ 2015, 3, e708. [CrossRef]

45. Lawrence, M.A.; Chin, A.; Swanson, B.T. Biomechanical Comparison of the Reverse Hyperextension Machine and the Hyperextension Exercise. J. Strength Cond. Res. 2019, 33, 2053-2056. [CrossRef] [PubMed]

46. Kim, J.-W.; Han, J.-Y.; Kang, M.-H.; Ha, S.-M.; Oh, J.-S. Comparison of Posterior Oblique Sling Activity during Hip Extension in the Prone Position on the Floor and on a Round Foam Roll. J. Phys. Ther. Sci. 2013, 25, 977-979. [CrossRef] [PubMed]

47. Jeon, I.; Kwon, O.; Weon, J.-H.; Hwang, U.; Jung, S.-H. Comparison of Hip- and Back-Muscle Activity and Pelvic Compensation in Healthy Subjects During 3 Different Prone Table Hip-Extension Exercises. J. Sport Rehabil. 2017, 26, 216-222. [CrossRef]

48. Kawama, R.; Takahashi, K.; Wakahara, T. Effect of Hip Joint Position on Electromyographic Activity of the Individual Hamstring Muscles During Stiff-Leg Deadlift. J. Strength Cond. Res. 2021, 35, S38-S43. [CrossRef] [PubMed] 
49. Ryu, Y.; Roh, H. Cervical, Trunk, and Lower Extremity Muscle Activities during Bridging Exercise on Stable vs. Unstable Bases of Support. J. Phys. Ther. Sci. 2012, 24, 585-588. [CrossRef]

50. Choi, K.; Bak, J.; Cho, M.; Chung, Y. The effects of performing a one-legged bridge with hip abduction and use of a sling on trunk and lower extremity muscle activation in healthy adults. J. Phys. Ther. Sci. 2016, 28, 2625-2628. [CrossRef]

51. Lee, J.-K.; Hwang, J.-H.; Kim, C.-M.; Lee, J.K.; Park, J.-W. Influence of muscle activation of posterior oblique sling from changes in activation of gluteus maximus from exercise of prone hip extension of normal adult male and female. J. Phys. Ther. Sci. 2019, 31, 166-169. [CrossRef]

52. Comfort, P.; Regan, A.; Herrington, L.; Thomas, C.; McMahon, J.; Jones, P. Lack of Effect of Ankle Position During the Nordic Curl on Muscle Activity of the Biceps Femoris and Medial Gastrocnemius. J. Sport Rehabil. 2017, 26, 202-207. [CrossRef]

53. Park, S.-Y.; Kim, S.-H.; Park, D.-J. Effect of slope angle on muscle activity during variations of the Nordic exercise. J. Exerc. Rehabil. 2019, 15, 832-838. [CrossRef]

54. Muyor, J.M.; Martín-Fuentes, I.; Rodríguez-Ridao, D.; Antequera-Vique, J.A. Electromyographic activity in the gluteus medius, gluteus maximus, biceps femoris, vastus lateralis, vastus medialis and rectus femoris during the Monopodal Squat, Forward Lunge and Lateral Step-Up exercises. PLoS ONE 2020, 15, e0230841. [CrossRef] [PubMed]

55. Jónasson, G.; Helgason, A.; Ingvarsson, P.; Kristjánsson, A.M.; Briem, K. The Effect of Tibial Rotation on the Contribution of Medial and Lateral Hamstrings During Isometric Knee Flexion. Sports Health A Multidiscip. Approach 2016, 8, 161-166. [CrossRef] [PubMed]

56. Park, S.; Yoo, W. Effects of hand and knee positions on muscular activity during trunk extension exercise with the Roman chair. $J$. Electromyogr. Kinesiol. 2014, 24, 972-976. [CrossRef]

57. Contreras, B.; Vigotsky, A.D.; Schoenfeld, B.J.; Beardsley, C.; Cronin, J. A Comparison of Gluteus Maximus, Biceps Femoris, and Vastus Lateralis Electromyography Amplitude in the Parallel, Full, and Front Squat Variations in Resistance-Trained Females. J. Appl. Biomech. 2016, 32, 16-22. [CrossRef] [PubMed]

58. Narouei, S.; Imai, A.; Akuzawa, H.; Hasebe, K.; Kaneoka, K. Hip and trunk muscles activity during nordic hamstring exercise. J. Exerc. Rehabil. 2018, 14, 231-238. [CrossRef]

59. Andersen, V.; Fimland, M.S.; Mo, D.-A.; Iversen, V.M.; Vederhus, T.; Rockland Hellebø, L.R.; Nordaune, K.I.; Saeterbakken, A.H Electromyographic Comparison of Barbell Deadlift, Hex Bar Deadlift, and Hip Thrust Exercises: A Cross-Over Study. J. Strength Cond. Res. 2018, 32, 587-593. [CrossRef] [PubMed]

60. Mjølsnes, R.; Arnason, A.; Østhagen, T.; Raastad, T.; Bahr, R. A 10-week randomized trial comparing eccentric vs. concentric hamstring strength training in well-trained soccer players. Scand. J. Med. Sci. Sports 2004, 14, 311-317. [CrossRef]

61. Freeman, B.W.; Young, W.B.; Talpey, S.W.; Smyth, A.M.; Pane, C.L.; Carlon, T.A. The effects of sprint training and the Nordic hamstring exercise on eccentric hamstring strength and sprint performance in adolescent athletes. J. Sports Med. Phys. Fitness 2019, 59, 1119-1125. [CrossRef]

62. Ribeiro-Alvares, J.B.; Marques, V.B.; Vaz, M.A.; Baroni, B.M. Four Weeks of Nordic Hamstring Exercise Reduce Muscle Injury Risk Factors in Young Adults. J. Strength Cond. Res. 2018, 32, 1254-1262. [CrossRef] [PubMed]

63. Seymore, K.D.; Domire, Z.J.; DeVita, P.; Rider, P.M.; Kulas, A.S. The effect of Nordic hamstring strength training on muscle architecture, stiffness, and strength. Eur. J. Appl. Physiol. 2017, 117, 943-953. [CrossRef]

64. Medeiros, D.M.; Marchiori, C.; Baroni, B.M. Effect of Nordic Hamstring Exercise Training on Knee Flexors Eccentric Strength and Fascicle Length: A Systematic Review and Meta-Analysis. J. Sport Rehabil. 2020, 30, 482-491. [CrossRef] [PubMed]

65. Van Dyk, N.; Behan, F.P.; Whiteley, R. Including the Nordic hamstring exercise in injury prevention programmes halves the rate of hamstring injuries: A systematic review and meta-analysis of 8459 athletes. Br. J. Sports Med. 2019, 53, 1362-1370. [CrossRef] [PubMed]

66. Al Attar, W.S.A.; Alshehri, M.A. A meta-analysis of meta-analyses of the effectiveness of FIFA injury prevention programs in soccer. Scand. J. Med. Sci. Sports 2019, 29, 1846-1855. [CrossRef] [PubMed]

67. Tyler, T.F.; Schmitt, B.M.; Nicholas, S.J.; McHugh, M.P. Rehabilitation After Hamstring-Strain Injury Emphasizing Eccentric Strengthening at Long Muscle Lengths: Results of Long-Term Follow-Up. J. Sport Rehabil. 2017, 26, 131-140. [CrossRef] [PubMed]

68. Van Dyk, N.; Bahr, R.; Whiteley, R.; Tol, J.L.; Kumar, B.D.; Hamilton, B.; Farooq, A.; Witvrouw, E. Hamstring and Quadriceps Isokinetic Strength Deficits Are Weak Risk Factors for Hamstring Strain Injuries. Am. J. Sports Med. 2016, 44, 1789-1795. [CrossRef]

69. Abdel-aziem, A.A.; Soliman, E.S.; Abdelraouf, O.R. Isokinetic peak torque and flexibility changes of the hamstring muscles after eccentric training: Trained versus untrained subjects. Acta Orthop. Traumatol. Turc. 2018, 52, 308-314. [CrossRef]

70. Vigotsky, A.D.; Halperin, I.; Lehman, G.J.; Trajano, G.S.; Vieira, T.M. Interpreting Signal Amplitudes in Surface Electromyography Studies in Sport and Rehabilitation Sciences. Front. Physiol. 2018, 8, 985. [CrossRef]

71. Chumanov, E.S.; Heiderscheit, B.C.; Thelen, D.G. The effect of speed and influence of individual muscles on hamstring mechanics during the swing phase of sprinting. J. Biomech. 2007, 40, 3555-3562. [CrossRef]

72. Novacheck, T.F. The biomechanics of running. Gait Posture 1998, 7, 77-95. [CrossRef]

73. Mendiguchia, J.; Conceição, F.; Edouard, P.; Fonseca, M.; Pereira, R.; Lopes, H.; Morin, J.-B.; Jiménez-Reyes, P. Sprint versus isolated eccentric training: Comparative effects on hamstring architecture and performance in soccer players. PLoS ONE 2020, 15, e0228283. [CrossRef] 